\begin{tabular}{|c|c|c|c|c|c|}
\hline MUNIBE Antropologia-Arkeologia & $n^{\circ} 72$ & $85-95$ & DONOSTIA & 2021 & ISSN 1132-2217 • eISSN 2172-4555 \\
\hline
\end{tabular}

\title{
El abrigo de Haza La Viña: un yacimiento de arte rupestre esquemático en el valle del río Sorbe (Muriel, Guadalajara, España)
}

\author{
The Haza La Viña rock shelter: a Schematic rock art site \\ in the Sorbe River Valley (Muriel, Guadalajara, Spain)
}

PALABRAS CLAVES: Arte-Esquemático, Sistema Central, Pintura, Cazoletas, Prehistoria reciente. GAKO-HITZAK: Arte-Eskematikoa, Erdialdeko Sistema, atzeko euskarriak, Historiaurre hurbila. KEY WORDS: Schemathic art, Iberian Central System, Paintings, Cup-marks, Late Prehistory.

\section{Ignacio TRIGUERO(1), Luis LUQUE(1), Samuel CASTILLO-JIMÉNEZ(1), Manuel ALCARAZ-CASTAÑO(1)}

\section{RESUMEN}

Se presenta un nuevo yacimiento de arte rupestre esquemático en el valle del río Sorbe, junto a la población de Muriel (Tamajón, Guadalajara). Se localiza en el interior de una covacha abovedada contigua a una cavidad de mayores dimensiones y contiene un único panel con un total de 6 unidades gráficas: cuatro antropomorfos y un signo en pintura roja en torno a dos cazoletas parcialmente superpuestas. En el centro de la península ibérica, junto a los grandes conjuntos esquemáticos de Valonsadero (Soria) y el Duratón (Segovia), se comienza a conformar un tercer grupo en la vertiente sur del sistema Central, del cual Haza la Viña constituye uno de sus ejemplos mejor conservados. Asimismo, la aparente sincronía de las representaciones permite ahondar en los esquemas compositivos de este horizonte gráfico, cuya cronología situamos en el Neolítico-Calcolítico. Se trata del único conjunto rupestre prehistórico conservado en la actualidad en este valle.

\section{LABURPENA}

Sorbe ibaiaren haranean, Muriel herriaren (Tamajón, Guadalajara) alboan, dagoen labar-arte eskematikoko aztarnategi berriari buruzko azalpena da. Neurri handiagoa duen barrunbe baten alboko ganga-formako haitzulo baten barruan dago eta guztira 6 unitate grafiko dituen panel bakarra jasotzen du: lau antropomorfo eta partzialki gainjarrita dauden bi atzeko euskarriren inguruan pintura gorriz egindako ikur bat. Iberiar penintsularen erdian, Valonsaderoko (Soria) eta Duratóngo (Segovia) multzo eskematiko nagusien alboan, hirugarren talde bat osatzen hasi da Erdialdeko Sistemako hegoaldean eta Haza de Viña da ondoen zainduta dagoen adibideetako bat. Era berean, irudietan itxuraz topa dezakegun sinkroniak horizonte grafiko hau osatzen duten eskemak sakontzea ahalbidetzen digu eta haien kronologia Neolitoan-Kalkolitoan koka dezakegu. Gaur egun bailara honetan topa dezakegun historiaurreko labar-multzo bakarra da.

\section{ABSTRACT}

We report a new site with Schematic rock art in the Sorbe River Valley, next to the town of Muriel (Tamajón, Guadalajara, Spain). It is located inside a vaulted cave next to a larger cavity and contains a single panel bearing 6 graphic units: four anthropomorphic figures and a sign in red paint around two partially superimposed cup-marks. In the center of the Iberian Peninsula, together with the well-known major clusters of Schematic rock art sites of Barranco del Duratón (Segovia) and Valonsadero (Soria), both on the northern Meseta, a third group to south of the Iberian Central System is emerging. The Haza la Viña rock shelter shows one of the best preserved rock art panels of this southern cluster. Its figures are clearly identified, and their homogeneity allows us to define the panel as an intentional composition. Despite the difficulties of dating, the site is best described as a Neolithic-Chalcolithic composition based on its thematic and stylistic features. Moreover, Haza la Viña has the added value of being the only rock art site in the Sorbe valley and the easternmost of the Spanish Central System group.

\footnotetext{
(1) Área de Prehistoria. Universidad de Alcalá. C/ Colegios 2, 28800. Alcalá de Henares. Autores: Ignacio Triguero: C/ Ledancas 5, $4^{\circ} \mathrm{A}, 19400$ Brihuega (Guadalajara): Ignaciotriguero@gmail.com. https:/orcid.org/0000-0003-4685-0134, Luis Luque: https:/orcid.org/0000-0001-56953036, Samuel Castillo-Jiménez: https:/orcid.org/0000-0003-3042-8940, Manuel Alcaraz-Castaño: https:/orcid.org/0000-0001-6291-9512
} 


\section{INTRODUCCIÓN}

El centro de la península ibérica se revela hoy como una zona con una acumulación de conjuntos gráficos pospaleolíticos que contrasta con la imagen que ofrecía hace unas décadas (Lucas, 2006: 29). En la vertiente sur del sistema Central (Alcolea-González et al., 1993), han sido determinantes las prospecciones de la banda caliza situada en las inmediaciones de la confluencia de los ríos Jarama y Lozoya (Alcolea-González et al., 1992). Sin embargo, las evidencias con las que contamos se derivan aún hoy de un registro parcial del territorio (Jiménez y Barroso, 1995; Lancharro y Bueno, 2017), en el que observamos importantes vacíos en áreas potencialmente relevantes para comprender los patrones de movilidad y asentamiento en el territorio de las sociedades prehistóricas. El valle del Sorbe es un ejemplo de este conocimiento fragmentado. A pesar de los escasos $10 \mathrm{~km}$ que separan este río del vecino Jarama en sus cursos altos, y de que ambos valles comparten entidad y paisaje, hasta ahora el primero carecía de arte pospaleolítico, a excepción del abrigo perdido de Las Quintillas (Ortego, 1979) y el covacho del Ocejón, en la ladera del mismo monte, prácticamente en la divisoria entre ambas cuencas (Sebastián Caudet y Gómez-Barrera, 2003). El abrigo de Las Quintillas, situado a una distancia de 2 km de Haza la Viña, desapareció presumiblemente bajo las aguas del embalse de Beleña, construido en 1982.

El Jarama y sus arroyos subsidiarios, por su parte, cuentan con una decena de cuevas y abrigos decora- dos publicados. El hallazgo del abrigo de Haza la Viña amplía hacia el este la extensión del núcleo artístico y propone relaciones con otras áreas geográficas. Además, la situación atípica en el interior de una cavidad a la que apenas llega la luz diurna y la homogeneidad estilística apuntan a una composición sincrónica con una intencionalidad única, características que amplían la casuística de estas representaciones.

\section{LOCALIZACIÓN Y AMBIENTE}

En julio de 2020, parte de nuestro equipo de investigación participaba en trabajos de prospección geoarqueológica en los alrededores de Muriel (Guadalajara). Los objetivos de dichos trabajos se centraban en localizar nuevos yacimientos y áreas de captación relacionables con la ocupación del valle del Sorbe durante el Pleistoceno superior (Alcaraz-Castaño et al., 2017; 2019; 2021), así como en la caracterización geomorfológica y datación de las terrazas fluviales de dicho valle. En este contexto se produjo el hallazgo de una pequeña cavidad con un panel decorado de estilo esquemático hasta ahora inédito.

El abrigo, denominado "Haza La Viña", se encuentra en un relieve calcáreo de unos 50 a 75 m de altura y alargado en dirección noreste-suroeste, situado en la margen izquierda del cauce del río Sorbe. Este relieve configura una cresta divisoria entre el Sorbe y el arroyo de Jócar, en las cercanías de la población de Muriel (870 m s.n.m.), Guadalajara (Fig. 1). Sus coordenadas UTM son: X: 484039,85 Y: 4536831,73 (Fig.1).

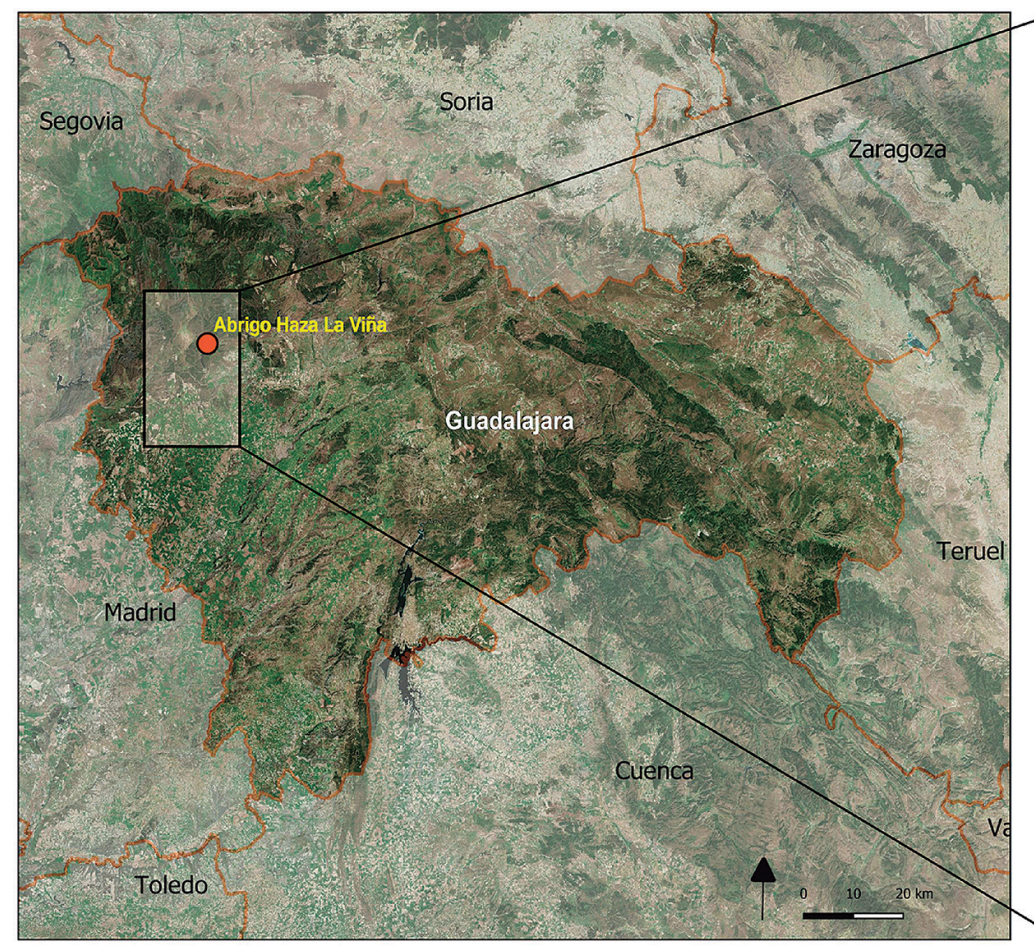

Fig.1. Localización del abrigo de Haza la Viña. / Location of the Haza la Viña rock shelter.

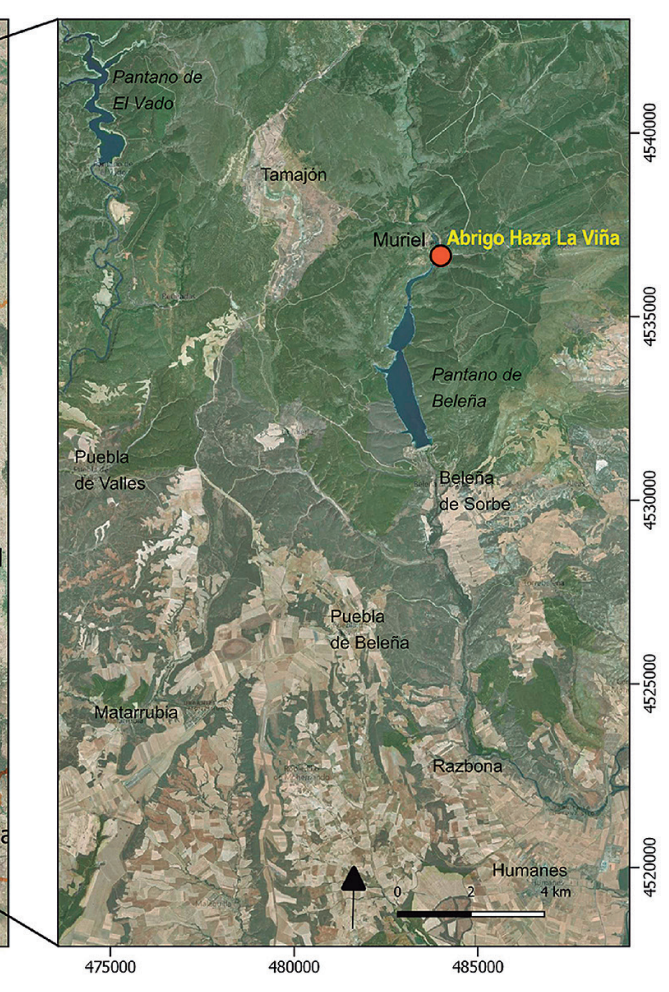


La formación del abrigo se relaciona con la karstificación cuaternaria de un estrecho conjunto de dolomías y calizas dolomíticas de edad Cretácico superior que se engloba en la llamada orla caliza del sistema Central, ya que se sitúa en sus márgenes norte y sur. En la zona de estudio, la orla limita por una parte con los relieves de rocas paleozoicas y triásicas de la sierra de Ayllón al norte y noroeste, y por otra con el relleno sedimentario terciario de la cuenca del Tajo, más concretamente de la cuenca de Madrid, al este y sur.

El río Sorbe pertenece a la cuenca hidrográfica del Tajo, tiene una longitud de $71 \mathrm{~km}$ y atraviesa una cuenca de 472 km2 de superficie hasta su desembocadura en el río Henares. El abrigo de Haza la Viña se sitúa en su tramo medio, a unos $20 \mathrm{~km}$ de la desembocadura y todavía en una zona relativamente encajada del valle, pero donde éste comienza a ensancharse y formar un paisaje más abierto. La cavidad se encuentra a una cota de 897 m sobre el nivel del mar, a 9 m por debajo de la cresta rocosa en ese punto y a unos $61 \mathrm{~m}$ sobre el cauce actual del río Sorbe (Fig.2). La desembocadura de un arroyo que bordea el relieve calizo en la margen opuesta, así como el depósito de un abanico aluvial y una terraza fluvial $a+5 \mathrm{~m}$, permitieron la formación de una superficie de escasa pendiente frente al abrigo que, durante buena parte del Holoceno, sería activa como llanura de inundación del río. De hecho, la estimación de incisión de los valles de las cuencas atlánticas (Silva et al., 2016) sugiere un encajamiento del cauce de entre 0,5 y 2,0 m por encima del actual en los últimos 10.000 años.

El paisaje actual se encuentra intensamente antropizado, con numerosas áreas de cultivo (muchas de ellas abandonadas en las últimas décadas), una intensa repoblación forestal de pinares y la inundación de parte del valle por el pantano de Beleña, lo que aporta pocas referencias sobre la vegetación que se desarrolló durante los últimos miles de años. Si utilizamos el Mapa de Series de Vegetación de España (Rivas-Martínez, 1987), que representa la comunidad botánica que existiría en un área dada sin influencia antrópica, observamos que la zona estaría dominada por tres series de vegetación: robledales de melojos (Quercus pyrenaica) en las partes altas del valle, encinares de Quercus rotundifolia o encina (Quercus ilex) en el curso medio y estos encinares acompañados por quejigares (Quercus faginea) en las zonas más bajas y, de forma más frecuente, en el vecino valle del Henares en el que desemboca (Fig.3).

La visibilidad de los yacimientos de arte rupestre pospaleolítico ha sido objeto de recientes investigaciones para determinar la implantación de los grupos en el territorio, lo que incluye redes de comunicación e intercambio y zonas de captación de recursos (Wheat-

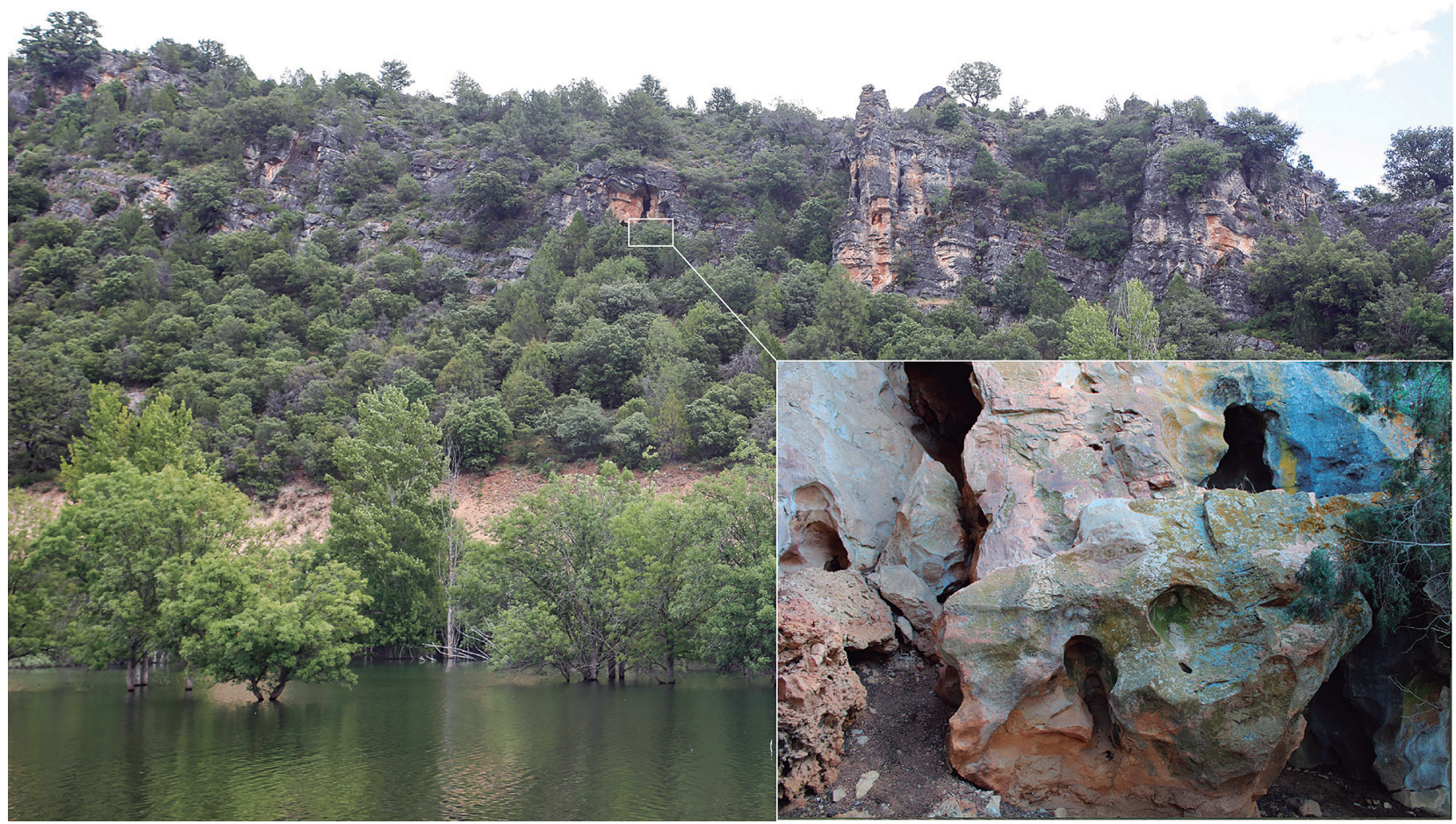

Fig.2. Vista frontal del yacimiento. A la derecha, el abrigo de Haza la Viña, a la izquierda, la cavidad contigua. / Front view of the site. On the right side, Haza la Viña rock shelter, on the left, adjacent cave. 


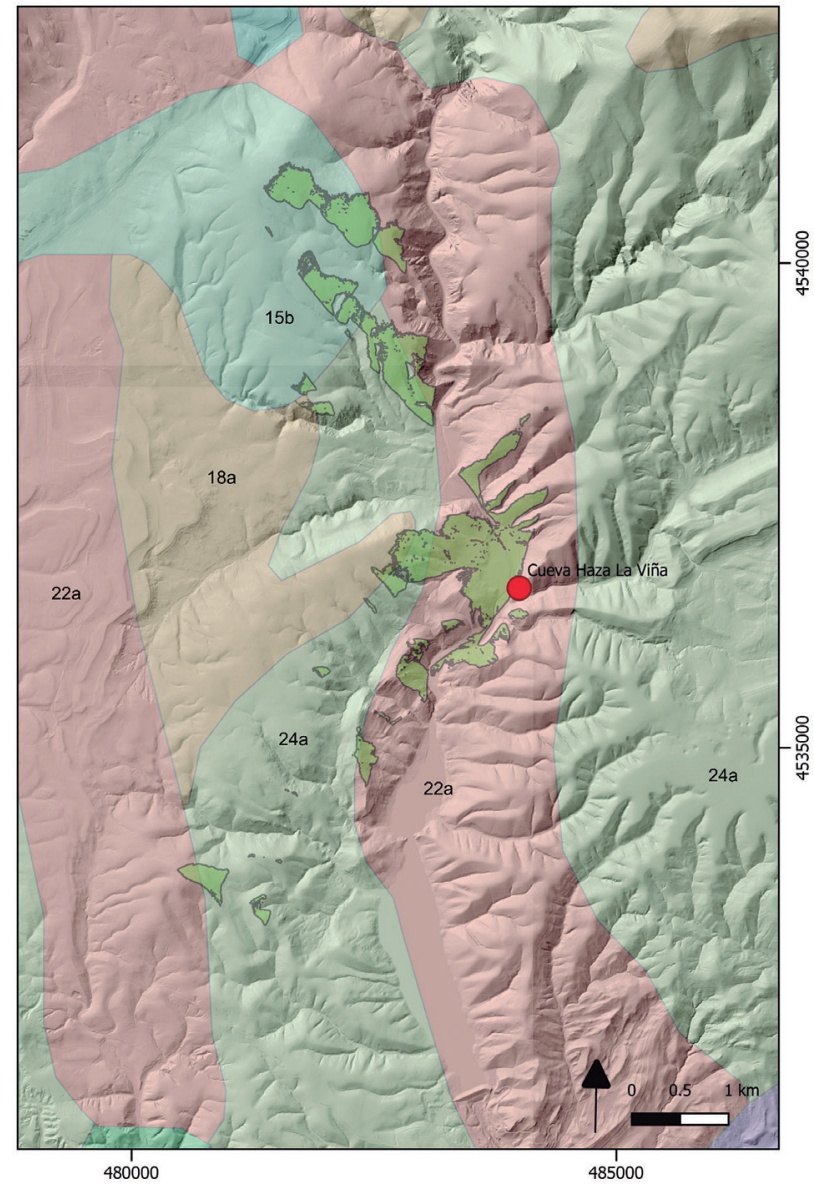

Fig.3. Localización del abrigo de Haza La Viña y el alto valle del Sorbe en el Mapa de Series de vegetación de España. Códigos: 15b Juniperus thurifera, 18a Quercus faginea, 22a Quercus rotundifolia con Quercus faginea y $24 a \mathrm{Q}$. rotundifolia. En color verde se representa la visibilidad desde el abrigo, calculada mediante SIG. / Location of the Haza La Viña shelter and the upper Sorbe valley in the Vegetation Series Map of Spain. Codes: 15b Juniperus thurifera, 18a Quercus faginea, 22a Quercus rotundifolia with Quercus faginea and $24 \mathrm{a} Q$. rotundifolia. The green color represents the visibility from the shelter, calculated by GIS.

ley, 1995; Fairén, 2002). El análisis obtenido mediante el SIG (QGIS 3.4) desde el punto donde se ubica el abrigo, muestra que la visual cubre las laderas meridionales (solanas) de unos $5 \mathrm{~km}$ aguas arriba del valle del Sorbe y, más escasamente, las laderas norte aguas abajo, en una franja relativamente estrecha. No obstante, esta visual cubre completamente la desembocadura del mencionado arroyo lateral y terraza junto al cauce del Sorbe, que probablemente estaría cubierta por vegetación de ribera y herbáceas. Respecto a las áreas forestadas, la zona que abarca esta visual presenta una mayor diversidad que la descrita anteriormente, añadiendo a los encinares, melojares y quejigares los sabinares de sabina albar (Juniperus thurifera).

\section{DESCRIPCIÓN DE LA CAVIDAD}

El abrigo (fig.4) está formado por una cavidad con dos pequeños espacios de sección aproximadamen- te elíptica y techo cónico, de unos 2,20 m de altura, conectados y separados por un pequeño puente de roca. La cavidad se formó por la disolución de la roca bajo condiciones freáticas del karst, como muestran las numerosas huellas cóncavas de erosión del agua en sus paredes y la formación de estructuras circulares. El abrigo, con 2,2 $\mathrm{m}$ de profundidad y una anchura máxima de 1,9 m, se encuentra a escasos metros de otra cavidad mayor con la que no está conectado en la actualidad. Ambas tienen un desarrollo condicionado por fracturas verticales en la roca y la disolución ha dado lugar a tubos y pasillos de morfología irregular y orientación dominantemente sureste, aproximadamente perpendicular al valle. La cavidad mayor presenta restos de rellenos sedimentarios endokársticos, como brechas de clastos angulosos de dolomía en una matriz areno-arcillosa y limos arcillosos laminados que se encuentran como parches muy erosionados en las paredes. Éstos se encuentran parcialmente cubiertos por finas coladas de espeleotema de forma botroidal. A pesar de la escasa distancia que separa esta cavidad del abrigo de Haza La Viña, este último carece de depósitos similares o espeleotemas antiguos y solamente muestra huellas de erosión por el flujo de agua sobre la roca madre.

El sustrato sobre el que se han aplicado las pinturas está constituido por dolomías de edad Santoniense-Campaniense (Cretácico superior) de la transición entre las formaciones Calizas del Burgo de Osma, típicamente representadas al suroeste, y la Formación Dolomías de Somolinos, situada al noreste (Barea et al., 2002, Gil et al., 2004 y 2010). Se trata de calcarenitas dolomíticas muy compactas y cementadas, con una estratificación bien definida, algo tableadas, que se estructuran en varias secuencias estrato decrecientes formadas por capas de entre 1,2 y 0,2 m de potencia. Las dolomías tienen aspecto masivo, excepto por trazas de bioturbación y una laminación difusa en algunos casos, debido a que la dolomitización ha borrado las estructuras sedimentarias previas, lo que las hace más homogéneas. Las capas buzan entre 8 y 140 al sureste por la deformación del sustrato, que ha sido elevado tectónicamente hacia el noroeste durante la Orogenia Alpina, dando lugar a un relieve en cuestas o en "hogback" de los carbonatos mesozoicos alrededor de los materiales pizarrosos y cuarcíticos del Paleozoico y a los depósitos detríticos de las facies Buntsandstein, más antiguos, que constituyen el núcleo del Sistema Central en esta región (Portero et al., 1990). La

La topografía de las cavidades ha sido realizada utilizando un distanciómetro láser Disto X2 de la marca Leica. Este aparato ha permitido medir distancias (error $2 \mathrm{~mm}$ ), rumbo e inclinación (error de 0,5) a partir de distintas estaciones de referencia desde el exterior de la cavidad hasta las zonas más profundas accesibles, acumulando más de 160 puntos georeferenciados que han permitido construir con precisión la cartografía. 


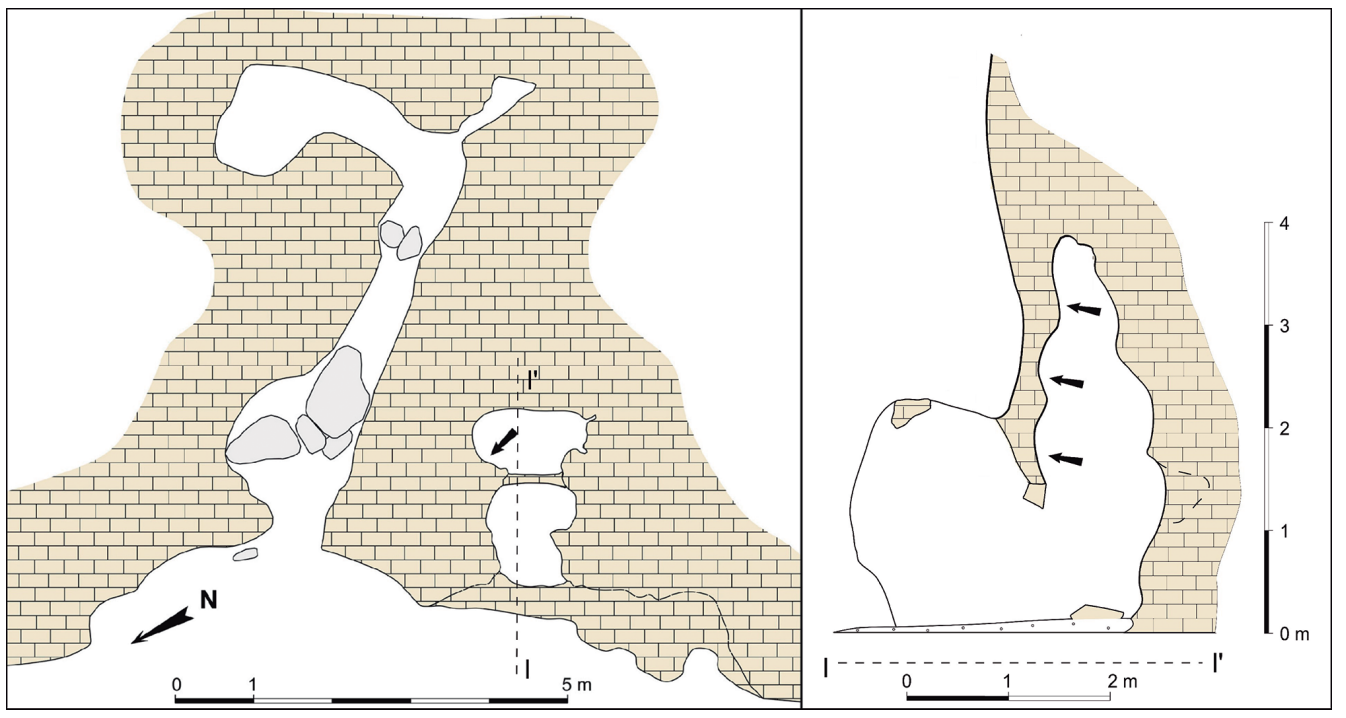

Fig.4. Topografía aproximada del abrigo de Haza La Viña y la cavidad lateral con la situación del Panel 1. Approximate topography of the Haza La Viña shelter and the lateral cavity with the location of Panel 1.

\section{METODOLOGÍA DE REGISTRO}

La configuración del espacio decorado condiciona la documentación gráfica debido a la escasez de luz y la estrechez del sitio, que no permite una fotografía completa del panel que contiene las decoraciones. Por ello, se ha optado por obtener un ortomosaico mediante fotogrametría con el objetivo de mostrar la totalidad de las grafías en su conjunto. Para la iluminación se han empleado dos focos led de tonalidad blanca y para el registro gráfico una cámara Réflex Canon EOS2000D. Tras tomar una veintena de imágenes en formato .raw, se ha generado una malla material de la que se ha extraído el ortomosaico final mediante el software Agisoft Metashape $®$. Sobre esta imagen se ha realizado el calco del panel (Fig.5). Las fotografías de detalle de cada figura no han necesitado este procedimiento. En lugar de realizar dibujo arqueológico, se han mantenido las formas originales con color aumentado mediante Adobe Photoshop $\AA$ (Fig.6). Para comprobar la presencia de pigmento no perceptible a simple vista, se ha utilizado el filtro Camera Raw de Adobe Photoshop $®$, aislando las tonalidades de los pigmentos del resto.

Para la descripción de los motivos rupestres se ha utilizado como base la clasificación clásica de Acosta (1968), sobre la que se han introducido las modificaciones pertinentes de cara a su adecuación al registro documentado en Haza la Viña.

\section{RESULTADOS: DESCRIPCIÓN DE LAS GRAFÍAS}

Las grafías se distribuyen en un único panel (Fig.5), que se encuentra en la pared izquierda del abrigo, a $160 \mathrm{~cm}$ del suelo, y presenta unas dimensiones de 127 $\mathrm{cm}$ de largo x $55 \mathrm{~cm}$ de ancho. El centro de la composición lo ocupan dos cazoletas superpuestas flanqueadas por dos antropomorfos, con un tercero situado sobre las tres unidades gráficas anteriores. En la parte superior del panel, un cuadrúpedo o pectiniforme se sitúa distanciado del resto de figuras. Finalmente, un cuarto antropomorfo muy perdido cierra el panel en su parte inferior. A excepción de las cazoletas, el resto de figuras se han realizado en pintura. La descripción pormenorizada de los motivos es la siguiente.

1. Antropomorfo en rojo, posiblemente acéfalo y con extremidades en " $v$ " invertida. Es la figura peor conservada del conjunto a causa de los óxidos que ocupan la pared, por lo que puede que su acefalia sea producto de su mal estado de conservación. Técnica: trazo lineal único. Tipología: figura humana esquemática: piernas en ángulo (Acosta, 1983: 18). Alt.: $12 \mathrm{~cm}$. Anch.: $7,5 \mathrm{~cm}$.

2. Antropomorfo sexuado en rojo, de cuerpo marcadamente alargado y extremidades en arco. Técnica: trazo lineal único Tipología figura humana esquemática, con tren superior de tipo golondrina (Acosta, 1968: 27, Fig.9) Alt.: $16 \mathrm{~cm}$. Anch.: 6,5 cm.

3. Dos cazoletas superpuestas en las que se aprecian marcas de piqueteado y posterior abrasionado. Alt.: 3,5 cm. Anch.: 6,5 cm.

4. Antropomorfo en rojo anaranjado, posiblemente sexuado, de cuerpo alargado y brazos en arco. Su parte inferior se encuentra deteriorada por alteraciones biológicas inducidas por la filtración de agua. Técnica: trazo lineal único. Tipología figura humana esquemática, cercana al tipo golondrina. (Acosta, 1968: 27, Fig.9) Alt.: $12 \mathrm{~cm}$. Anch.: $5 \mathrm{~cm}$.

5. Antropomorfo sexuado en color rojo. Cuerpo alargado y extremidades en arco. Es el antropomorfo de mayores dimensiones, ocupando un lugar central y elevado en el conjunto. Se aprecia una línea que parte de su brazo derecho que puede ser interpretada bien como una extensión inidentificable de la figura bien como un simple trazo residual. 


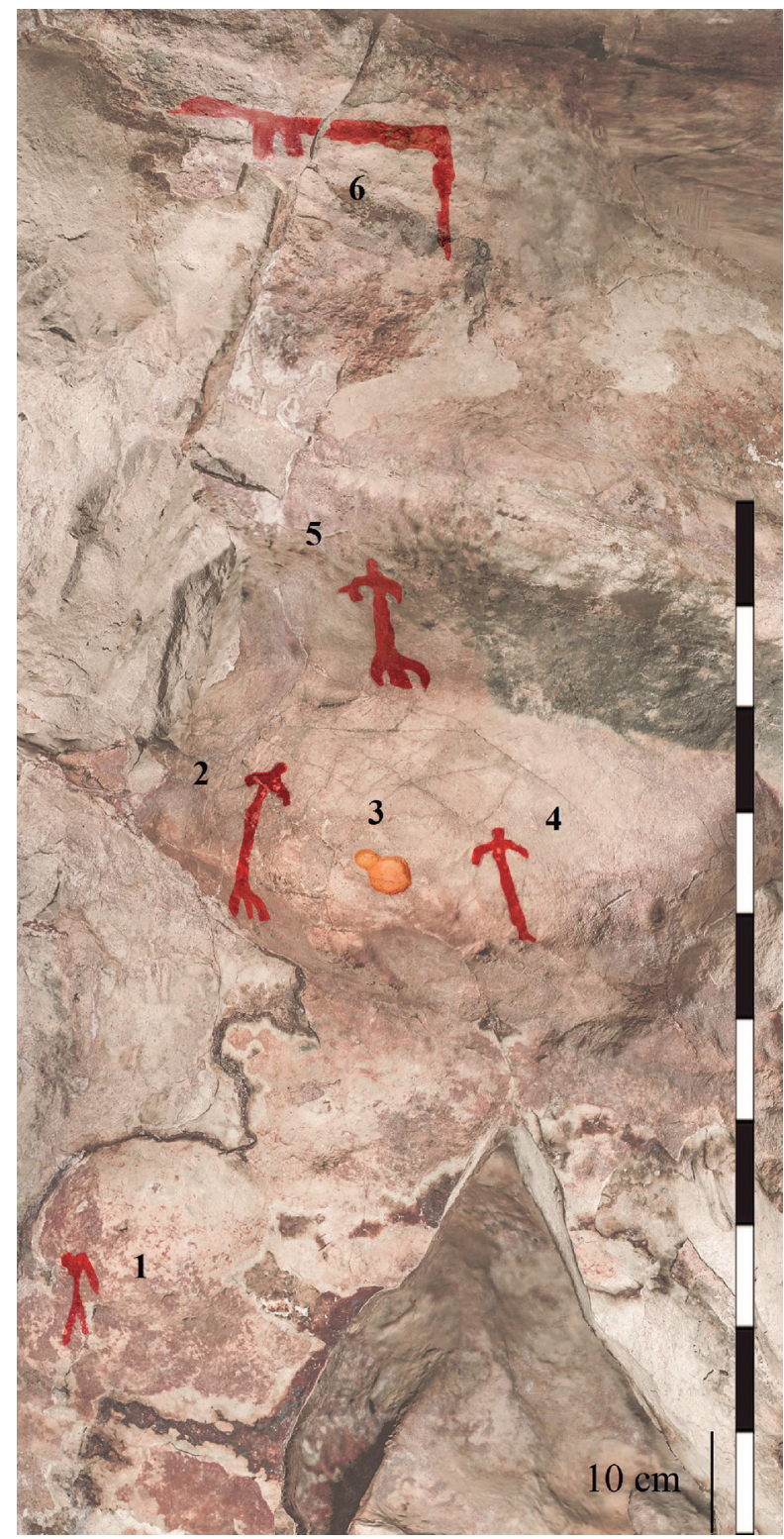

Fig.5. Calco del panel 1. / Panel 1 tracing.

Técnica: trazo lineal único Tipología figura humana esquemática con tren superior tipo golondrina que quizá fuese brazos en asa. (Acosta, 1968: 27, Fig.9) Alt.: $15 \mathrm{~cm}$. Anch.: $7 \mathrm{~cm}$.

6. Cuadrúpedo/pectiniforme en color rojo. La parte izquierda de la figura se encuentra perdida, en parte por una rotura, así como por alteraciones químicas provocadas por la filtración de agua. Se compone de un trazo horizontal con una gran expansión vertical en su extremo derecho y dos de menor tamaño en su parte central. Técnica: trazo lineal único. Hemos optado por clasificarlo dentro de la forma cuadrúpedo (Acosta, 1968: 55, Fig.8), si bien su morfología podría ser asimilada dentro del grupo de los pectiniformes Alt.: $12 \mathrm{~cm}$. Anch.: $29 \mathrm{~cm}$.
Para visualizar el 3D del panel que contiene las grafías pulse aquí: https://skfb.ly/oqpVz

\section{DISCUSIÓN: MARCO CULTURAL Y CRONO- LÓGICO}

Las estribaciones meridionales del sistema Central cuentan con un nutrido grupo de estaciones rupestres pospaleolíticas (Alcolea-González et al., 1993). Este territorio ocupa, a grandes rasgos, las cabeceras y cursos altos de los ríos Manzanares, Lozoya, Jarama y Sorbe, con especial concentración en su parte central (Fig 7).

Por su denominación geográfica, el grupo de la Vertiente Sur del sistema Central debería incluir los yacimientos más orientales de la Comunidad de Madrid, el abrigo de La Enfermería (Jiménez, 1992) y el Cerro de San Esteban (Pastor, 1997), pertenecientes a Pelayos de la Presa y San Martín de Valdeiglesias respectivamente. Sin embargo, han sido excluidos en la bibliografía previa de este grupo debido a su desconexión con el resto de yacimientos. No consideramos problemática la omisión, puesto que nuestro grupo se encuentra más cercano a otros núcleos como el del Duratón que a estos yacimientos orientales.

El emplazamiento de los abrigos esquemáticos del área que nos ocupa responde en mayor medida a criterios geológicos y topográficos que a patrones de preeminencia sobre el paisaje. Se encuentran, en su mayoría, en superficies calcáreas iluminadas naturalmente que afloran en laderas poco accidentadas. El campo visual desde la boca de Haza la Viña cubre las laderas y cumbres aguas arriba y abajo del río Sorbe. Dentro de la misma formación rocosa, no es el abrigo con mayor campo visual ni su cavidad contigua la de mayores dimensiones de la zona. Por ello, la hipótesis de preeminencia territorial de Haza la Viña cuenta con evidencia limitada o, en el mejor de los casos, la misma evidencia que tendría por sus posibilidades poblacionales. La elección de Haza la Viña para un fin simbólico debe responder a otras razones que, a falta de una intervención arqueológica más amplia, no nos son accesibles.

Haza la Viña amplía el grupo del centro de la Península al este de manera sensible, con una continuidad estilística más palpable que la que ofrecía el desparecido abrigo de las Quintillas (Ortego, 1979). Además, presenta una homogeneidad interna evidente. La morfología, tamaño y coloración de los antropomorfos es muy similar, a excepción, quizá, del antropomorfo de extremidades en " $v$ " invertida (motivo 1). Este cuenta con paralelos formales cercanos, en el covacho del Ocejón (Anciones et al., 1993) o en el gran antropomorfo del Arroyo de la Vega (Alcolea-González et al., 1993) (Fig 8: 1,2). El resto (motivos 2, 4 y 5), de tronco alargado y extremidades en arco, se asemejan a los presentes en los sitios madrileños de Los Horcajos (García-Valero, 1995) o Los Aljibes (Priego, 1991) (Fig 8: 3,4). Fuera del ámbito más cercano, compar- 

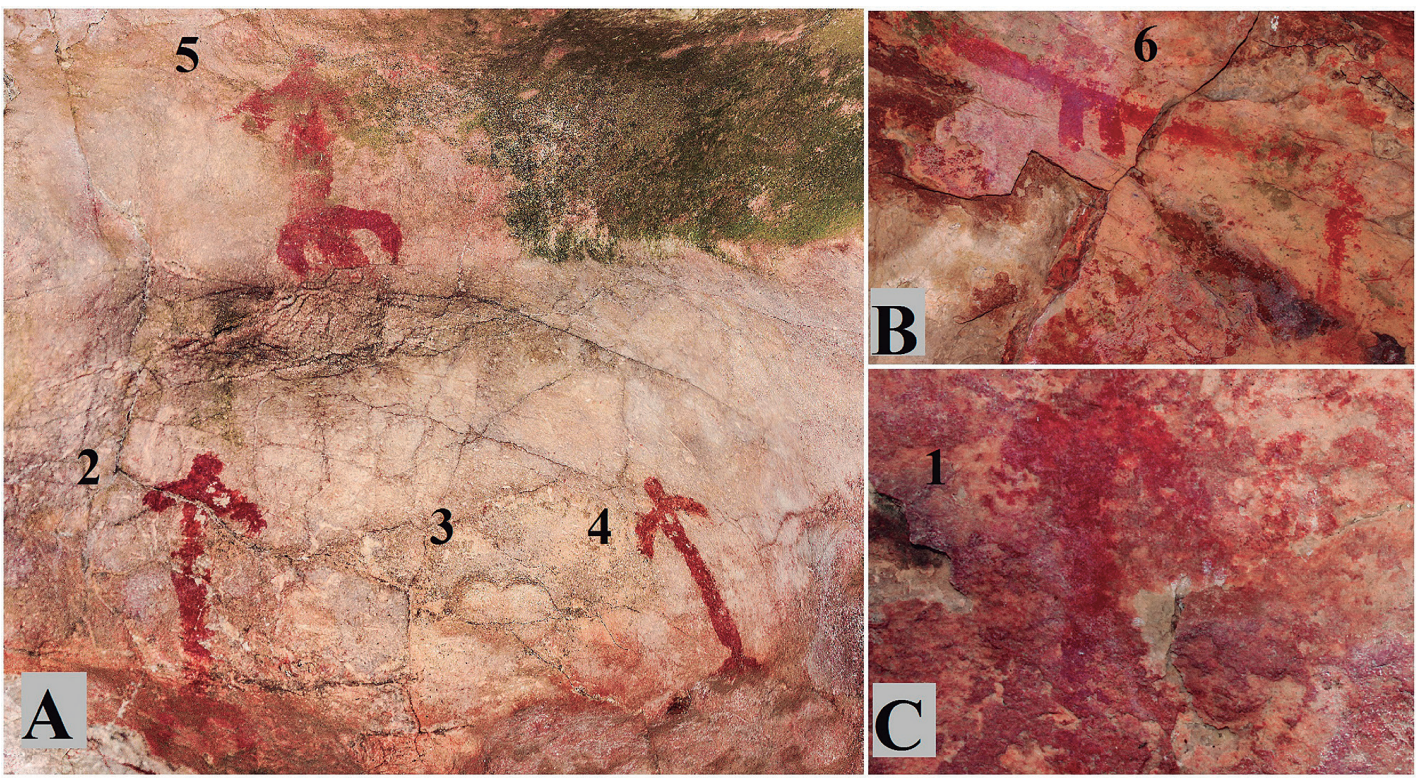

Fig.6. Detalle de las grafías documentadas en el abrigo de Haza la Viña. Colores modificados mediante Adobe Photoshop. / Detail of the figures documented on the Haza la Viña shelter. Color modification using Adobe Photoshop.

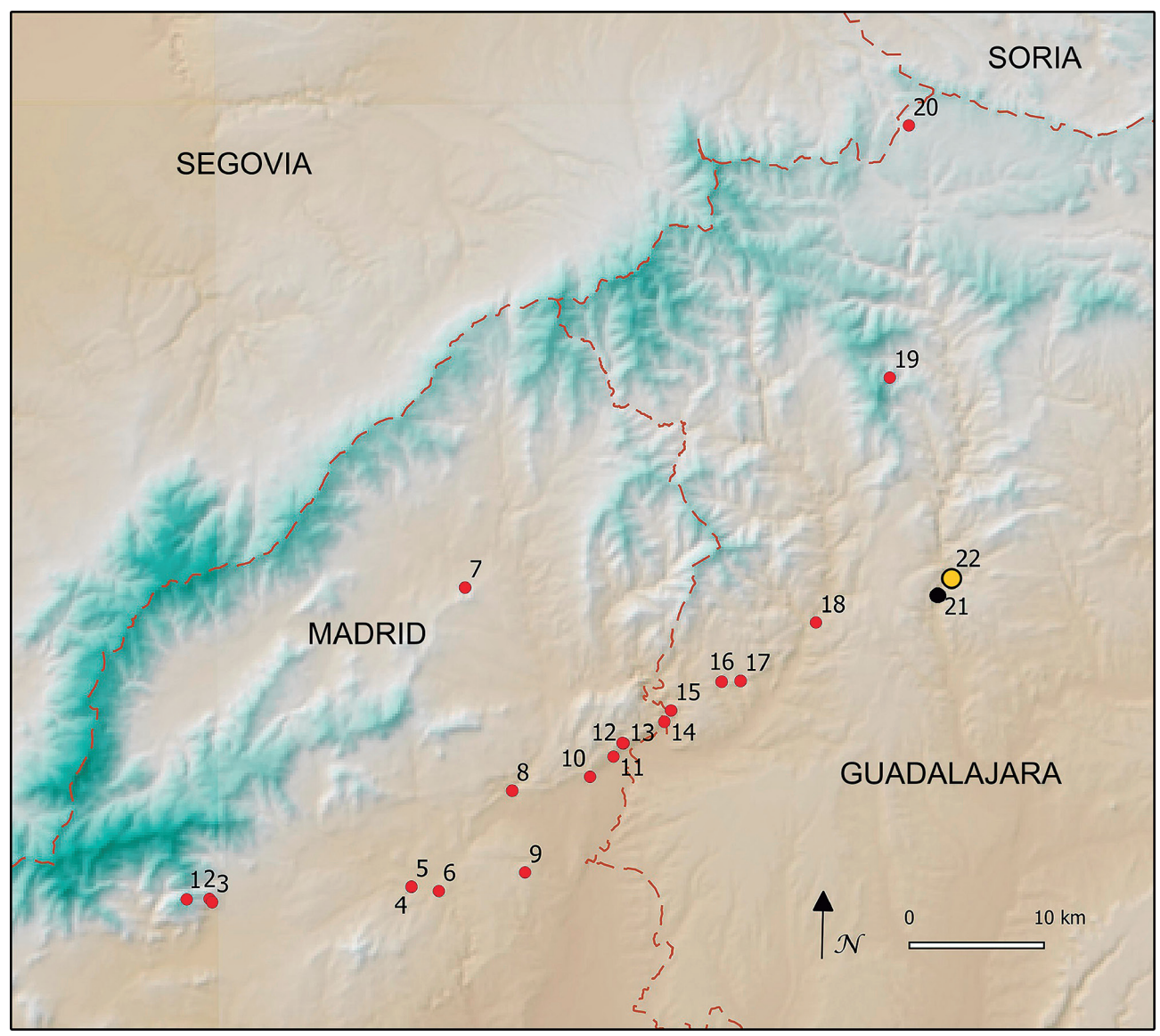

Fig.7. Yacimientos de arte esquemático en la vertiente sur del sistema Central. / Schematic rock art sites on the southern slope of the Spanish Central System. 1- Abrigo 82/55-3R, 2- Abrigo 82/54-2R (Lucas et al., 2006), 3- Los Aljibes (Priego, 1991), en Manzanares el Real; 4- Cueva del Quejigal (Jiménez, 2002) 5- Abrigo de Valdesalices (Lucas et al., 2006), 6- Los Alcores (Lucas et al., 2006) en Guadalix de la Sierra; 7-La Dehesa (Pastor-Muñoz, 1997) en Buitrago de Lozoya; 8Cueva del Derrumbe (Lucas et al., 2006) en Torrelaguna; 9- Abrigo de Los Horcajos (García-Valero, 1995) en EI Vellón; 10- Abrigo de Belén (Alcolea-González et al. 1994) en Torremocha de Jarama; 11- cueva del Aire (Fernández-Posse, 1980; Lucas et al., 2006); 12- cueva de Las Avispas (Alcolea-González et al., 1992), 13- Abrigo del Pollo (Antona, 1987; Lucas et al., 2006); 14- Covacho del Pontón de la Oliva (Lucas et al., 2006), en Patones; 15- cueva del Sumidero (Jiménez, 1997), 16- cueva de Los Hombres, 17- cueva del Arroyo de la Vega, (Alcolea-González et al., 1993) en Valdepeñas de la Sierra; 18- cueva de las Ovejas (Balbín, 2002) en Valdesotos; 19- Barranco del Reloje /Covacho del Ocejón (Anciones, 1993; Sebastián Caudet y Gómez Barrera, 2003) en Valverde de los Arroyos: 20- El Portalón (Ortego, 1963; Gómez-Barrera 1996) en Villacadima; 21- abrigo de Las Quintillas (Ortego 1979), en Muriel (desaparecido/ lost); $22-$ Haza la Viña. 
ten notables similitudes con los presentes en el Abrigo Remacha en la Hoz del Duratón (Cardito y de Andrés, 2011) o algunos motivos de la Lastra, Peñascales III (Gómez-Barrera, 2001) o Cueva Grande, con una disposición gráfica más cercana a Haza la Viña (Gómez Barrera, 1982: 171; fig. 73) (Fig 8: 7).

A pesar de estas similitudes formales, para encontrar asociaciones análogas debemos dirigirnos al sur peninsular Destacan varias escenas del Peñón de la Virgen (Martínez-García, 1984), por técnica, estilo y composición (Fig 8: 5,6). De modo más general las Palomas IV (Breuil y Burkitt, 1929: Fig. XVIII) o Peña Escrita (Góngora, 1868), ofrecen paneles donde los antropomorfos comparten escena con cuadrúpedos de características similares al motivo 6 de Haza la Viña. Esta última figura tiene un diseño original, ya que la posición de dos extremidades juntas en su parte media lo aleja de los ejemplos típicos, aunque esta variabilidad es habitual en las representaciones de cuadrúpedos, tal como se viene señalando desde antiguo (Breuil, 1929: 9).

La presencia de cazoletas (motivo 3) como punto central de la composición tampoco es común. Cuando comparten espacio con los paneles pintados no aparecen integradas en la composición, sino ocupando su propio espacio, como sucede en el relativamente cercano yacimiento de la sierra de Caldereros (Lancharro y Bueno, 2017). De igual modo, su asociación con antropomorfos es poco frecuente en el ámbito subterráneo, aunque con expresivos ejemplos, como en la cueva de Santa Cruz (Soria) (Gómez-Barrera 1992) y más habitual en el arte megalítico, de forma evidente en los dólmenes extremeños de la Vega del Guadancil o Huerta de las Monjas (Bueno y Balbín, 2003).

La interpretación cronológica de las pinturas del abrigo de Haza La Viña genera los problemas habituales de este tipo de representaciones: dificultad de realizar dataciones directas y ausencia de materiales arqueológicos asociados. La socorrida cerámica simbólica proporciona antropomorfos desde el Neolítico en Cova de l'Or (García-Borja et al., 2011), Cova de la Sarsa (Pérez Botí, 2001), Abric de la Falguera (Molina y García-Borja, 2006), el incompleto de Atapuerca (Alday et al., 2019) o los de cronología más dudosa de la cueva del Agua en Prado Negro (Navarrete y Capel, 1977), entre otros. El Calcolítico supone una cierta disrupción en la representación más esquematizada del motivo, copado por las versiones oculadas, bitriangulares o naturalistas en cerámica y soportes muebles (Martín y Camalich, 1982). En el Bronce de la Meseta destaca el antropomorfo de la Galería del Sílex, con su homólogo parietal (Apellániz y Domingo, 1987) y en el Hierro I el del madrileño Camino de las Cárcavas (Almagro-Gorbea et al., 1996). Si bien, tipológicamente los más cercanos a los que nos ocupan son los neolíticos de Cueva del Agua y Atapuerca, el motivo antropomorfo es, por su esquematismo y variada casuística, difícil de atribuir con claridad a una época determinada. Sin embargo, recientes excavaciones insisten en la relación entre composiciones afines a Haza la Viña y hábitats neolíticos (Vega et al., 2021). Del mismo modo, los zoomorfos demuestran la persistencia de formas esquematizadas desde el Neolítico antiguo en la zona mediterránea (Torregrosa y Galiana, 2001: 162-163), y,

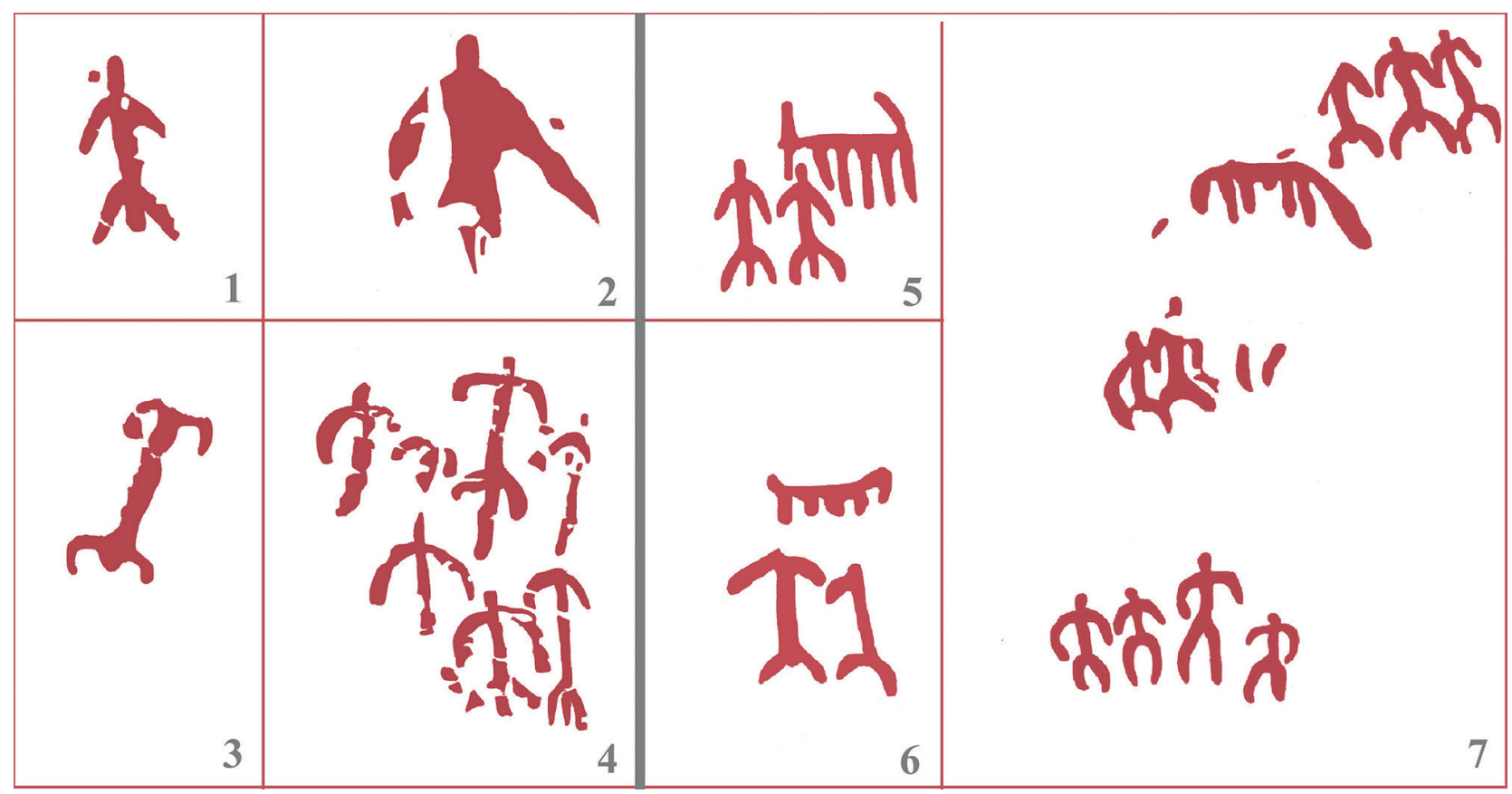

Fig.8. Paralelos estilísticos y compositivos del abrigo de Haza la Viña. Modificado por I. Triguero. / Sites stylistically related to Haza la Viña, modified by I. Triguero: 1: Covacho del Ocejón (Sebastián Caudet y Gómez-Barrera, 2003); 2: Arroyo de la Vega (Alcolea González et al., 1993) 3: Los Horcajos (García-Valero, 1995); 4: Los Aljibes (Priego, 1991): 5,6: Peñón de la Virgen de Gilma (Martínez-García 1984); 7: Cueva Grande (Gómez Barrera, 1982: 171; fig. 73). 
ya en el Interior peninsular, durante el Calcolítico (Blasco y Baena, 1996), Edad del Bronce y primer Hierro (Rodanés y Royo, 1986), con perduración en el Hierro II (Azcárraga et al., 2012).

Si atendemos al poblamiento prehistórico de la zona vemos que, en un radio de unos $20 \mathrm{~km}$, hay constancia de yacimientos pertenecientes a cada uno de los periodos de la Prehistoria reciente. Para el eje entre las poblaciones de Torrelaguna y Tamajón se ha propuesto la existencia de una entidad cultural importante que parte del Neolítico final y se interna en el Calcolítico pre-campaniforme (Alcolea-González et al., 1993; Rubio, 2006: 282). Dentro de este espacio destaca el fenómeno funerario en cueva del alto Jarama (Jiménez y Barroso, 1995; Alcolea-González et al., 1993), interpretado como reflejo local del fenómeno megalítico regional (Bueno et al., 2002). Algunas de estas cavidades presentan inhumaciones colectivas de diferente cronología, entre las que destacan El Destete (Jiménez y Alcolea-González, 2001), Jarama II (Jordá y Mestres, 1999) y, sobre todo, El Rebollosillo (Díaz del Río et al., 2017), con una amplia batería de dataciones que constatan la gran variabilidad en las prácticas funerarias para la segunda mitad del III milenio A.C. en la Meseta. La relación entre enterramientos humanos y grafías es habitual en varios de estos yacimientos, ya sea por coincidencia directa, como en las cuevas de Las Avispas y El Aire, o por proximidad. como en la cueva del Arroyo de la Vega.

En cuanto a la realidad arqueológica más inmediata, los yacimientos pertenecientes a la Prehistoria reciente más cercanos a Haza la Viña son las cuevas de la Vaca -a 500m- (Jiménez y Barroso, 2000) y el Gorgocil (Ortego, 1979), ambos con cerámica del Bronce medio. A mayor distancia se encuentra el yacimiento del Morro, con materiales que abarcan desde el Bronce hasta la romanización (Valiente, 1992b; Barroso, 1993). Finalmente, en la vecina población de Cogolludo se encuentra la Loma del Lomo, hábitat de grandes dimensiones de la Edad del Bronce (Valiente, 1992a). En todo caso, la posibilidad de relación entre estos yacimientos y Haza la Viña es meramente especulativa, dada la ausencia de evidencias arqueológicas en el abrigo y la amplia cronología del poblamiento prehistórico de la zona.

\section{CONCLUSIONES}

El yacimiento de Haza la Viña es, actualmente, el único yacimiento de arte rupestre prehistórico conservado en el valle del río Sorbe y el segundo de su cuenca, junto con el Covacho del Ocejón. Teniendo en cuenta el escaso trabajo de campo realizado en este valle en comparación con el vecino valle del Jarama, es probable que la excepcionalidad de este hallazgo se corrija en el futuro cercano si se realizan prospecciones sistemáticas. Del mismo modo, la conservación de los pigmentos los hace susceptibles a análisis de autoría de mayor calado (Martínez-Sevilla et al. 2020).
Los motivos rupestres registrados, reconocibles con pocas reservas como pertenecientes al ciclo gráfico esquemático, presentan sin embargo evidentes problemas en cuanto a su adscripción cronológica. Ello obedece no solo a la ausencia de registro arqueológico asociado y la imposibilidad de datación directa de los pigmentos, sino a problemas para establecer una periodización detallada del fenómeno rupestre esquemático tanto a escala peninsular como regional (vid. Hernández-Pérez, 2009). No obstante, la hipótesis de relación cronológica y poblacional entre el arte rupestre esquemático de los altos valles de Jarama y Sorbe con el fenómeno funerario neolítico-calcolítico regional resulta la más sólida según la evidencia actual. Esto se debe, en primer lugar, a la abundancia de cavidades con contextos funerarios para este periodo y, en segundo lugar, a la coincidencia, aunque escasa, de enterramientos asociados a grafías rupestres en el vecino valle del Jarama.

\section{AGRADECIMIENTOS}

Este trabajo se enmarca en el proyecto de investigación "Population dynamics and cultural adaptations of the last Neandertals and first Modern humans in inland Iberia: a multi-proxy investigation" (MULTIPALEOIBERIA) financiado por el Consejo Europeo de Investigación (ERC-2018-STG-805478, IP Manuel Alcaraz-Castaño). También agradecemos a la Fundación Banco Sabadell por el apoyo financiero a la investigación predoctoral del primer firmante.

Los trabajos de prospección geoarqueológica que permitieron el hallazgo del abrigo de Haza La Viña fueron autorizados por la Consejería de Educación, Cultura y Deportes de la Junta de Castilla-La Mancha (Expediente 20.1029). Agradecemos su participación al resto de miembros del equipo de prospección y en especial a los profesores José Javier Alcolea González por sus apreciaciones a una versión previa del manuscrito y a Primitiva Bueno Ramírez por su asesoramiento.

\section{BIBLIOGRAFÍA}

Acosta, P., 1968. La pintura esquemática en España. Universidad de Salamanca.

Acosta, P., 1983. Técnica, estilo, temática y tipología en la pintura rupestre esquemática hispana. Zéphyrus XXXVI, 13-25.

Alcaraz-Castaño M., Alcolea-González J., Balbín-Behrmann R. de, Kehl M., Weniger G.-C., 2019. Recurrent human occupations in Central Iberia around the Last Glacial Maximum. The Solutrean sequence of Peña Capón updated. In: Schmidt, I., Cascalheira, J. (eds.), Human adaptations to the Last Glacial Maximum: The Solutrean and its neighbors, 148-170. Cambridge Scholars Publishing.

Alcaraz-Castaño, M., Alcolea-González J., Weniger, G.C., Álvarez-Figueras, I., Arteaga,A., Baena-Preysler, J., de Balbín-Behrmann, R., Yravedra, J., 2017. Neandertales y humanos modernos en Guadalajara. Boletín de la Asociación de Amigos del Museo de Guadalajara 8, 13-44. 
Alcaraz-Castaño M., Alcolea-González J.J., Andrés-Herrero M., Castillo-Jiménez S., Cuenca-Bescós G., Cuartero F., Kehl M., López-Sáez J.A., Luque L., Pérez-Díaz S., Piqué R., Ruiz-Alonso M., Weniger G.-C., Yravedra, J., 2021. First modern human settlement recorded in the Iberian hinterland occurred during Heinrich Stadial 2 within harsh environmental conditions. Scientific Reports 11, 15161. https://doi.org/10.1038/ s41598-021-94408-w

Alcolea-Gonzalez J., Bunes-lbarra, F., García-Valero, M.A., Jiménez, P., 1993. Arte esquemático en la vertiente sur del sistema Central. Trabalhos de Antropología e Etnología 33, 199-216.

Alcolea-González, J., Baena J., García-Valero, M. A., Gómez-Hernanz, J., 1992. La Cueva de las Avispas, un yacimiento con Arte Rupestre en la Provincia de Madrid. Boletín de la Asociación de Amigos de la Arqueología 32, 19-22.

Alcolea-González, J., Bunes-lbarra, F., García-Valero, M. A., Márquez-Mora B. 1994. Las representaciones rupestres esquemáticas del Abrigo de Belén (Torremocha, Madrid). Estudios de Prehistoria y arqueología Madrileños 9, 29-32.

Alcolea-González, J., Bunes-lbarra, F., García-Valero, M. A., Gómez-Hernanz, J., Jiménez, P., 1993. Las pinturas rupestres esquemáticas de la cueva del Arroyo de la Vega (Valdepeñas de la Sierra). Wad-al-Hayara 20, 85-108.

Alday, A. Pérez-Romero, E. Iriarte, M. Francés-Negro, J.L. Arsuaga, J.M. 2019. Carretero Pottery with ramiform-anthropomorphic decoration from El Portalón de Cueva Mayor site (Sierra de Atapuerca, Burgos) and the globalized symbolic world of the first Neolithic. Quaternaty International 515, 125-137.

Almagro-Gorbea, M., López, L., Madrigal, A., Muñoz, K., Ramón Ortiz, J., 1996. Antropomorfo sobre cerámica de la I Edad del Hierro de la Meseta. Complutum 7, 141-146.

Anciones, R., Cardito, L. M., Ramírez, I., Etzel, E., 1993. Pinturas esquemáticas en "La Cueva" del Barranco del Reloje. Wad-al-Hayara 20, 109-126.

Antona, V., 1987. El Neolítico. 130 años de arqueología madrileña, 45-58. Comunidad de Madrid.

Apellániz, J. M., Domingo, S., 1987. Los materiales de superficie del santuario de la Galería del Sílex. In: Apellániz, J.M., Domingo, S. (eds.). Estudios sobre Atapuerca (Burgos) II, 1-278. Universidad de Deusto, Bilbao.

Azcárraga, S., Morín, J., Urbina, D., 2012. Conjunto cerámico de una estructura doméstica de Segunda Edad del Hierro en el yacimiento de La Guirnalda (Quer, Guadalajara). En: Morín, J., Urbina, D. (eds.), El Primer Milenio a. C. en la Meseta Central. De la Longhouse al Oppidum,vol. 2, II Edad del Hierro, 225-241. Madrid.

Barroso, R., 1993. El Bronce Final y la transición a la Edad del Hierro en Guadalajara. Wad-Al-Hayara 20, 9-44.

Barea, J., López Martínez, J., Durán, J.J., 2002. Desarrollo del karst versus litoestratigrafía en los bordes norte y sur del Sistema Central español. Boletín Geológico y Minero 113(2), 155-164.

Blasco, M. C., Baena, J., 1996. El yacimiento de Las Carolinas y la cerámica simbólica campaniforme. Algunos datos para su interpretación. En: Moure, A. (ed.), El Hombre Fósil 80 años después, 417-446. Universidad de Cantabria. Santander

Breuil, H., Burkitt, M., 1929. Rock paintings of Southern Andalusia. A description of a Neolithic and Copper Age art group. Clarendon Press, Oxford.
Bueno, P., Barroso, R., Jiménez, P., 2002. Culturas productoras y metalúrgicas en la provincia de Guadalajara: Estado de la cuestión. En: García-Soto, E., García, M.A. (Eds.), Actas del primer Simposio de Arqueología de Guadalajara. Homenaje a Encarnación Cabré, 229-276. Sigüenza.

Bueno P., Balbín, R., 2003. Grafías y territorios megalíticos en Extremadura. En: Gonçalves, V.S. (ed.), Muita gente, poucas antas? Origens, espaços e contextos do Megalitismo, Actas do II Colóquio Internacional sobre Megalitismo, 407-448. Instituto Português de Arqueologia (Trabalhos de Arqueologia 25).

Cardito, L. M., Andrés-Herrero, M. de, 2011. La estación de arte rupestre esquemático Abrigo Remacha en el barranco del río Duratón (Segovia). Férvedes 7, 97-103.

Díaz del Río, P., Consuegra, S., Audije, J., Zapata, S., Cambra, O., González, A., Waterman, A., Tykot, R., 2017. Un enterramiento del III milenio AC en el centro de la Península Ibérica: El Rebollosillo (Torrrelaguna, Madrid). Trabajos de Prehistoria 74(1), 68-85. https://doi.org/10.3989/tp.2017.12184

Fairén, S., 2002. Visibilidad y percepción del entorno, análisis de la distribución del arte rupestre esquemático mediante Sistemas de Información Geográfica. Lucentum XXI-XXII, 27-43.

Fernández-Posse, M. D., 1980. Los materiales de la Cueva del Aire de Patones (Madrid). Noticiario Arqueológico Hispánico 10, 41-64.

García Borja, P., Cortell, E., Pardo S., Pérez Jordà, G., 2011. Las cerámicas de la Cova de l'Or (Beniarrés, Alacant). Tipología y decoración de las colecciones del Museu d'Alcoi, Recerques del Museu d'Alcoi 20, 71-138.

García-Valero, M.A., 1995. Las pinturas rupestres esquemáticas del Abrigo de los Horcajos (El Vellón, Madrid). Estudios de Prehistoria y Arqueología Madrileñas 10, 7-13.

Gil, J., Carenas, B., Segura, M., García Hidalgo, J.F., García, A., 2004. Revisión y correlación de las unidades litoestratigráficas del Cretácico Superior en la región central y oriental de España. Revista de la Sociedad Geológica de España 17(3-4), 249-266.

Gil, J., García-Hidalgo, J.F., Segura, M., López Olmedo, F., García, A., Díaz de Neira, J.A., Montes, M., Nozal, F., 2010. El Cretácico del Sistema Central (España): Registro estratigráfico, contexto deposicional y esquema evolutivo. Boletín de la Real Sociedad Española de Historia Natural, Sección Geología 104, 15-36.

Gómez-Barrera, J. A., 1992. Grabados rupestres postpaleolíticos del Alto Duero. Serie de Investigación del Museo Numantino 1, 93-101.

Gómez-Barrera, J. A., 1996. El abrigo de 'El Portalón' (Villacadima, Guadalajara): nuevos calcos de sus pinturas y una propuesta para su protección y conservación. Wad-al-Hayara 23, 39-69.

Gómez-Barrera, J.A., 2001. Pinturas rupestres de Valonsadero y su entorno. Caja Rural de Soria, Soria.

Góngora, M., 1868. Antigüedades prehistóricas de Andalucía. C. Moro, Madrid.

Hernández-Pérez, M., 2009. Acerca del origen del arte esquemático. Tabona 17, 63-92.

Jiménez, J. 1997. El abrigo del Sumidero: nueva estación esquemática en Guadalajara. Kalathos 16, 7-18.

Jiménez, C., 1992. Estudio preliminar: las pinturas rupestres esquemáticas de La Enfermería. Pelayos de la Presa (Madrid), Arqueología, Paleontología y Etnografía 3, 7-30. Comunidad de Madrid. 
Jiménez, P., Barroso, R., 1995. El fenómeno funerario durante la Prehistoria Reciente en el centro de la Meseta: la provincia de Guadalajara. Trabalhos de Antropología e Etnología 35(2), 211-223.

Jiménez P. J., Barroso, R. M., 2000. Diversificación del hábitat del Bronce Medio en Guadalajara: la Cueva de La Vaca. Actas do $3^{\circ}$ Congresso de Arqueologia Peninsular V, 131-144.

Jiménez P., Alcolea-González, J., 2001. Excavaciones arqueológicas en la cueva del Destete (Valdepeñas de la Sierra, Guadalajara) Cuestiones preliminares. Actas del Primer simposio de Arqueología de Guadalajara, Vol II, 293-308.

Jordá, J.F., Mestres, J.S., 1999. El enterramiento calcolítico precampaniforme de Jarama II: una nueva fecha radiocarbónica para la prehistoria reciente de Guadalajara y su integración en la cronología de la región. Zephyrus 52, 175-190.

Lancharro, M. A., Bueno, P., 2017. Pintura Esquemática y territorios de la prehistoria reciente en la cuenca interior del Tajo. Zephyrus 80, 33-47.

López Escudero, A., 2002. Un arqueólogo descubre en Guadalix una cueva con pinturas de hace 5.000 años. El País, 20 de enero de 2002 https://elpais.com/diario/2002/01/20/madrid/1011529463_850215.html (consulta 25-01-2021).

Lucas, M.R., 2006. Acercamiento a legos, versados en prehistoria y gestores del patrimonio de la Comunidad de Madrid. In: Lucas, M.R., Cardito, L.M., Gómez, J. (coords.), Dibujos en la roca. El arte rupestre en la Comunidad de Madrid, 23-30. Consejería de Cultura y Deportes de la CAM.

Lucas, M. R., Cardito L. M., Gómez-Hernánz, J., 2006. Inventario de yacimientos. In: Lucas, M.R., Cardito, L.M., Gómez, J. (coords.), Dibujos en la roca. El arte rupestre en la Comunidad de Madrid, 151-262. Consejería de Cultura y Deportes de la CAM.

Martín, D., Camalich, M.D., 1982. La "cerámica simbólica" y su problemática (aproximación a través de los materiales de la colección L. Siret). Cuadernos de Prehistoria de la Universidad de Granada 7, 267-306.

Martínez-García, J., 1984. El Peñón de la Virgen: un conjunto de pinturas rupestres en Gilma (Nacimiento, Almería). Asociaciones recurrentes, simbolismo y modelo de distribución. Cuadernos de Prehistoria de la Universidad de Granada 9, 39-84.

Martínez-Sevilla, F., Arqués, M., Jordana, X., Malgosa, A., Rodríguez, J. A. L., Romero, M. S., Sharpe, K., Rus, J. C., 2020. Who painted that? The authorship of Schematic rock art at the Los Machos rockshelter in southern Iberia. Antiquity 94 (377), 1133-1151. https://doi.org/10.15184/aqy.2020.140.

Molina, L., García-Borja, P., 2006. Vas amb decoració simbòlica al'Abric de la Falguera. En: García Puchol, O., Aura, J.E. (eds), El Abric de la Falguera (Alcoi, Ala-cant). 8000 años de ocupación humana en la cabe-cera del río de Alcoi, 189-211. Ajuntament d'Alcoi, Dipt. Provincial d'Alacant, C.A.M.

Navarrete, M. S., Capel, J., 1977. La cueva del Agua de Prado Negro (Iznalloz, Granada). Cuadernos de Prehistoria de la Universidad de Granada 2, 19-62.

Ortego, T., 1963. Las pinturas rupestres del Portalón en el término de Villacadima. Ampurias XXV, 207-216.

Ortego, T., 1979. Un nuevo abrigo con pinturas rupestres en el término de Muriel (Guadalajara). Actas del XV Congreso Nacional de Arqueología (Lugo, 1977), 429-437.

Pastor, F. J., 1997. Nuevos hallazgos de arte rupestre esquemático en Madrid. Revista de Arqueología 192, 10-17.
Pérez Botí, G., 2001. La Cova de la Sarsa (Bocairent, Valencia). La decoración figurada de su cerámica neolítica. Una aproximación cronocultural. Recerques del Museu d'Alcoi 10, 43-58.

Portero, J.M., Aznar, J.M., Pérez González, A., González Lodeiro, F., 1990. Mapa geológico de España 1:50000, hoja $n^{\circ}$ 485 (Valdepeñas de la Sierra). IGME, Madrid.

Priego, C., 1991. Pinturas rupestres del abrigo de Los Aljibes en La Pedriza del Manzanares. Estudios de Prehistoria y Arqueología Madrileños 7, 87-123.

Rivas-Martínez, S., 1987. Mapa de Series de Vegetación de España. MAPAMA. Dirección General de Medio Natural y Política Forestal.

Rodanés, J.M., Royo, J.I., 1986. Representaciones zoomorfas del Bronce Final y Primera Edad del Hierro en el valle medio del Ebro. Estudios en Homenaje al Dr. Antonio Beltrán Martínez, 373-387. Universidad de Zaragoza.

Rubio, I., 2006. Pastores de ovejas y cultivadores de trigo: el color rojo domestica la naturaleza. Mirando las paredes. Contextualización cultural de los abrigos con Arte Postpaleolítico (Pintura Esquemática). Discusión Cronológica. In: Lucas, M.R., Cardito, L.M., Gómez, J. (coords.), Dibujos en la roca. El arte rupestre en la Comunidad de Madrid, 263-309. Consejería de Cultura y Deportes de la CAM.

Sebastián Caudet, A., Gómez-Barrera, J.A., 2003. Las pinturas rupestres esquemáticas del Covacho del Ocejón (Valverde de los Arroyos, Guadalajara). Saldvie 3, 1-13.

Silva, P.G., Roquero, E., López-Recio M., Huerta, P., Martínez-Graña, A., 2016. Chronology of fluvial terrace sequences for large Atlantic rivers in the Iberian Peninsula (Upper Tagus and Duero drainage basins, Central Spain). Quaternary Science Reviews 166, 188-203. https://doi.org/10.1016/j.quascirev.2016.05.027.

Torregrosa, P., Galiana, M. F., 2001. El Arte Esquemático del Levante Peninsular: una aproximación a su dimensión temporal. Millars XXIV, 111-155. Castellón.

Valiente, J. (Ed.), 1992b. La Celtización del Tajo Superior. Estudios de arqueología 3. Universidad de Alcalá de Henares.

Valiente, J., 1992a. La Loma del Lomo II. Cogolludo (Guadalajara). Patrimonio Historico-Arqueología Castilla La Mancha. Junta de Comunidades de Castilla La Mancha, Toledo.

Vega, S., Laborda, R, Lanau, P., Roda, X., González Marcén, P., Mora, R., Martínez-Moreno, J., 2021. Un enclave singular del Neolítico antiguo en el Prepirineo de Lleida: la Cova del Tabac (Camarasa, Lleida). Munibe Antropologia-Arkeologia 72 (Online first). https://doi.org/10.21630/maa.2021.72.03.

Wheatley, D., 1995. Cumulative viewshed analysis: a GIS based method of investigating intervisibility and its archaeological application. In: Lock, G., Stancic, Z., Archaeology and GIS: A European Perspective, 171-185. Taylor and Francis, London. 
